\title{
Correction to: The Chirality Theorem
}

\author{
José M. Gracia-Bondía, Jens Mund and Joseph C. Várilly
}

\section{Correction to: Ann. Henri Poincaré 19 (2018), 843-874 https://doi.org/10.1007/s00023-017-0637-3}

After formula (2.7a) of the paper, we omitted to indicate that its right-hand side, as a distribution in $p$, cannot be restricted to the zero-mass shell. Therefore, for massless particles, the two-point function is not well defined as it stands. On the other hand, the intertwiner function (2.3) is well defined and locally integrable, as stated. Thus, the corresponding string-local fields with light-like strings are hermitian form-valued distributions, rather than operatorvalued distributions.

As a consequence, a few expressions in Section 6 are merely formal. Nevertheless, all expressions for second-order tree-graph contributions to the $\mathbb{S}$ matrix elements or to interacting correlation functions are well defined for certain generic configurations of external momenta. Assuming that the Fourier transform of the switching function $g$ has support in a small region $\Delta$ around the origin, one can choose $l^{\perp}+\Delta$ away from all partial sums of the external momenta, thereby avoiding the singularities.

Furthermore, this issue does not affect the proofs or the conclusion of the article: in the arguments which determine the full set of couplings of the electroweak bosons to the fermions, that massless $A$-field appears only in a spectator role in Step 3-by then chirality of the charged bosons interactions has been demonstrated. In Step 4 there is a "crossing" between that field and the Maxwell field: this is entirely legitimate, since the corresponding two-point function is well-defined.

(It should also be noted that, in the massive case, the on-shell functions $M^{\varphi \chi}(p, l)$ should be regularized when they enter into time-ordered two-point

The original article can be found online at https://doi.org/10.1007/s00023-017-0637-3. 
functions (where $p$ is not restricted to the mass shell): each factor $(p l)$ must be replaced by $(p l)+i \varepsilon$ or $(p l)-i \varepsilon$, according as it stems from the $\chi$ - or the $\varphi$-intertwiner.)

We point out, finally, that there is a factor $\delta_{l}$ missing on the right hand sides of equations $(6.4 \mathrm{~b})$ and $(6.10 \mathrm{~b})$.

\section{Acknowledgements}

We thank Paweł Duch, Karl-Henning Rehren and Christian Gaß for friendly advice and helpful suggestions.

José M. Gracia-Bondía

Departamento de Física Teórica

Universidad de Zaragoza

50009 Zaragoza

Spain

e-mail: jmgb@unizar.es

Jens Mund

Departamento de Física

Universidade Federal de Juiz de Fora

Juiz de Fora MG 36036-900

Brasil

e-mail: mund@fisica.ufjf.br

Joseph C. Várilly

Escuela de Matemática

Universidad de Costa Rica

San José 11501

Costa Rica

e-mail: joseph.varilly@ucr.ac.cr 